\title{
The role of the state in model reduction with subspace and POD- based data-driven methods
}

\section{Conference Paper}

Author(s):

lannelli, Andrea (D); Smith, Roy (D)

Publication date:

2021

Permanent link:

https://doi.org/10.3929/ethz-b-000498452

Rights / license:

In Copyright - Non-Commercial Use Permitted

Originally published in:

https://doi.org/10.23919/ACC50511.2021.9482920

Funding acknowledgement:

178890 - Modeling, Identification and Control of Periodic Systems in Energy Applications (SNF) 


\title{
The role of the state in model reduction with subspace and POD-based data-driven methods
}

\author{
Andrea Iannelli and Roy S. Smith
}

presented at: 2021 American Control Conference,

DOI: 10.23919/ACC50511.2021.9482920, (O2021 IEEE.

\begin{abstract}
The paper investigates the selection of state sequences in data-driven projection-based model reduction methods that compute parsimonious models by forming regression problems featuring loworder fictitious states. Specifically, subspace identification and dynamic mode decomposition techniques are considered. It is shown that, while sharing a seemingly equivalent structure, they differ profoundly in the way these states are selected. A theoretical characterization of the differences is given, including a parametrization of a new class of state transformations implicitly used in both approaches and a balanced transformation obtained directly from data. Numerical examples are proposed to show the impact of these differences on the accuracy of the extracted low-order representations.
\end{abstract}

\section{INTRODUCTION}

Methods which enable models of dynamical systems to be constructed from data have traditionally been the focus of system identification [1], and are typically geared towards experimental practicalities (where e.g. data are noisy, and states are not measured) and control applications (which privilege particular model classes). Nonetheless, they share similar goals with methods developed in other communities for the identification of loworder structures from high-dimensional noise-free (often simulated) state trajectories, such as the pioneering works documented in [2] for the study of turbulence. The concept of Proper Orthogonal Decomposition (POD) in particular, revolving around the selection of a few modes capturing most of the energy in the data, boosted the research on approaches for computing models able to balance accuracy with complexity. A well known example is Dynamic Mode Decomposition (DMD) [3], which seeks to approximate the eigendecomposition of a low-order linear dynamics explaining the evolution of observed state sequences. Originally developed in the context of autonomous systems, the DMD framework has been extended to systems with input and output channels [4], [5], which makes it essentially an algorithm to obtain

This work is supported by the Swiss National Science Foundation under grant no. 200021_178890.

The authors are with the Department of Electrical Engineering, Automatic Control Lab, ETH, Zürich 8092, Switzerland iannelli/rsmith@control.ee.ethz.ch low-order state-space models given input, state projected onto the POD modes, and output trajectories.

A celebrated system identification technique that targets the same model class, but works only with input and (generally noisy) output data is Subspace Identification (SId) [6]. SId algorithms can all be interpreted as the identification of a subspace spanned by an extended observability matrix of the system, followed by the estimation of its state-space matrices [7]. Since the first step also reveals a basis for the state of the underlying system [8], one can recast the problem as a least-squares analogous to that solved in DMD. Despite this apparent similarity, a key question is which are the particular state sequences employed, since it is well known that linear states are only defined up to a similarity transformation. While this is inconsequential when the full model is identified, it becomes critical when seeking low-order representations by truncating the number of states used in the regression. This arbitrariness is further exacerbated by the fact that both problems employ fictitious states.

Motivated by these observations, which have not received much attention in the literature, the paper addresses the problem of extracting state sequences from noise-free data generated by linear dynamics for the purpose of obtaining low-order models. A partial reconciliation is first shown by introducing the concept of POD transformation, for which a parametrization is derived which analytically shows how one can place arbitrary singular values to any state with an appropriate similarity transformation. Then, a unique up-front (that is, not requiring knowledge of the state sequence) balanced transformation is presented to extract the SId states directly in balanced coordinates. This is markedly different from other approaches to data-driven balanced truncation such as Balanced POD (which requires the system's matrices and an adjoint model) and the Eigensystem Realization Algorithm [2] (which is a particular SId algorithm relying on realization theory, and does not provide the balancing transformation). The current work is related to [9], where a balanced state extraction was originally proposed, and [10], where improved algorithms for the identification of balanced state-space models were provided. In [6, Chapter 5] the importance of the state basis was also recognized, but POD-type bases, which 
seem relevant in view of the increasing popularity of related methods, were not considered.

Results are shown for a non-normal system (prototypical of certain types of dynamical models describing flows [11]) and a thermoacoustic model [12]. They both illustrate the importance of the state selection for the accuracy of low-order models and motivate the use of the projection strategies presented in the paper.

\section{BACKGROUND}

We consider a deterministic multi-input multi-output (MIMO) linear time-invariant (LTI) discrete-time system:

$$
\begin{aligned}
x_{t+1} & =A x_{t}+B u_{t}, \\
y_{t} & =C x_{t}+D u_{t},
\end{aligned}
$$

where $x \in \mathbb{R}^{n_{x}}$ is the state, $u \in \mathbb{R}^{n_{u}}$ is the input, and $y \in \mathbb{R}^{n_{y}}$ is the output, for which a lower order representation (i.e. with a number of states $r$ smaller than $n_{x}$ ) is sought. Useful related matrices are: reachability and observability Gramians $W_{r}=L_{r} L_{r}^{\top} \in \mathbb{R}^{n_{x} \times n_{x}}$ and $W_{o}=$ $L_{o} L_{o}^{\top} \in \mathbb{R}^{n_{x} \times n_{x}}$, and their Cholesky factors $L_{r}$ and $L_{o}$, respectively; the extended observability matrix $\Gamma_{l}=$ $\left[C^{\top}(C A)^{\top} \ldots\left(C A^{l-1}\right)^{\top}\right]^{\top} \in \mathbb{R}^{n_{y} l \times n_{x}}$, the reversed extended controllability matrix $\Omega_{l}=\left[A^{l-1} B \ldots A B B\right] \in$ $\mathbb{R}^{n_{x} \times n_{u} l}$; and the block Toeplitz matrix:

$$
\mathcal{T}_{l}=\left[\begin{array}{cccc}
D & 0 & 0 & 0 \\
C B & D & 0 & 0 \\
\vdots & \ddots & D & 0 \\
C A^{l-2} B & C A^{l-3} B & \cdots & D
\end{array}\right],
$$

Given an ensemble of vectors $z \in \mathbb{R}^{n_{z}}$, we denote by $z_{[i, j]}$ the matrix:

$$
z_{[i, j]}:=\left[\begin{array}{lll}
z_{i} \ldots & z_{j}
\end{array}\right] \in \mathbb{R}^{n_{z} \times(j-i+1)},
$$

and, when $z$ is a function of time, also the sequence $\left\{z_{k}\right\}_{k=i}^{j}$ of length $j-i+1$. The block Hankel matrix $\mathcal{H}_{l}\left(z_{[i, j]}\right)$ with depth $l$ associated with $z_{[i, j]}$ is:

$$
\mathcal{H}_{l}\left(z_{[i, j]}\right):=\left[\begin{array}{cccc}
z_{i} & z_{i+1} & \cdots & z_{j-l+1} \\
z_{i+1} & z_{i+2} & \cdots & z_{j-l+2} \\
\vdots & \vdots & & \vdots \\
z_{i+l-1} & z_{i+l} & \cdots & z_{j}
\end{array}\right]
$$

where $l$ is the number of block rows.

Given a matrix $X \in \mathbb{R}^{n \times m}$ with $n \leq m$, its SVD and QR factorizations are denoted by:

$$
\begin{aligned}
& X=U \Sigma V^{\top}, \\
& X=Q R,
\end{aligned}
$$

where $U \in \mathbb{R}^{n \times n}$, and $Q \in \mathbb{R}^{n \times n}$ are orthogonal matrices, $V \in \mathbb{R}^{m \times n}$ has orthonormal columns, $\Sigma \in \mathbb{R}^{n \times n}$ is diagonal, and $R \in \mathbb{R}^{n \times m}$ is upper trapezoidal.

The pseudo-inverse of $X$ is denoted by $X^{\dagger}$, while $X(:, i: j)$ and $X(:, j)$ denote the submatrix including the columns from $i$ to $j$ and the vector with column $j$, respectively.

\section{A. Subspace identification}

Subspace identification (SId) is a well established nonparametric technique to identify state-space MIMO models from input-output data [6]. While several algorithms have been proposed, the main idea revolves around two steps [7]. First, a subspace spanned by the columns of the extended observability matrix $\Gamma_{n_{x}+1}$ of the system is identified. Second, the state-space matrices are estimated. Inspired by realization theory, some methods first determine $A$ and $C$ by making use of the shift invariance property of the subspace spanned by $\Gamma_{n_{x}+1}$, and then $B$ and $D$, e.g. from $\mathcal{T}_{l}$. Alternatively, a state sequence can be extracted from the extended observability matrix and a least-squares problem solved for the state-space matrices. The latter approach is closely related to this work, and a brief summary of the algorithm in [8] is reported next.

Input and output trajectories of system (1), respectively $u_{[1, N]}$ and $y_{[1, N]}$, are used to build two block Hankel matrices:

$$
\begin{aligned}
H_{1,2 l}= & {\left[\begin{array}{l}
\mathcal{H}_{2 l}\left(u_{[1, N]}\right)(1: l,:) \\
\mathcal{H}_{2 l}\left(y_{[1, N]}\right)(1: l,:)
\end{array}\right], } \\
H_{2,2 l}= & {\left[\begin{array}{l}
\mathcal{H}_{2 l}\left(u_{[1, N]}\right)(l+1: 2 l,:) \\
\mathcal{H}_{2 l}\left(y_{[1, N]}\right)(l+1: 2 l,:)
\end{array}\right], }
\end{aligned}
$$

where $l$ is chosen such that $\ln _{y}$ (rows of the two output Hankel sub-matrices $\left.\mathcal{H}_{2 l}\left(y_{[1, N]}\right)\right)$ is greater than $n_{x}$ and smaller than $N-2 l+1$ (columns of the Hankel matrices). Under the assumption that the input is sufficiently exciting (i.e., that $\mathcal{H}_{2 l}\left(u_{[1, N]}\right)$ is full row rank), it holds that $\operatorname{rank}\left(H_{1,2 l}\right)=\operatorname{rank}\left(H_{2,2 l}\right)=l n_{u}+n_{x}$, i.e. the two sub-matrices of $\mathcal{H}_{2 l}\left(y_{[1, N]}\right)$ only add $n_{x}$ dimensions to the row space spanned by the corresponding input Hankel matrices. Moreover, $\operatorname{span}\left(H_{1,2 l}\right)$ $\cap \operatorname{span}\left(H_{2,2 l}\right)=\operatorname{span}\left(x_{[l+1, N-l+1]}\right)$ and thus any basis of this intersection gives a valid state vector sequence. This intersection is found in [8] via a double SVD. The first is used to find the orthogonal complement of $\left[\begin{array}{l}H_{1,2 l} \\ H_{2,2 l}\end{array}\right]$, providing a subspace of size $2 l n_{y}-n_{x}$ whose basis consists of $n_{x}$ vectors. A second SVD is employed to extract $\hat{x}_{[l+1, N-l+1]}$, whose row vectors provide one such basis.

The state-space identification problem is then formulated as the least-squares problem

$$
\min _{A, B, C, D}\left\|\left[\begin{array}{c}
\hat{x}_{[l+2, N-l+1]} \\
y_{[l+1, N-l]}
\end{array}\right]-\left[\begin{array}{cc}
A & B \\
C & D
\end{array}\right]\left[\begin{array}{c}
\hat{x}_{[l+1, N-l]} \\
u_{[l+1, N-l]}
\end{array}\right]\right\|_{F}^{2} .
$$

\section{B. POD and (IO)DMD(c)}

Proper Orthogonal Decomposition (POD) is a popular technique to extract low-order structures in highdimensional physical problems [2]. Consider $M$ members $\left\{w^{(k)}\right\}_{k=1}^{M}$ of a scalar field (the same reasoning applies to the multivariable case) $w: \mathbb{R} \rightarrow \mathbb{R}^{n}$. For example, $w$ can be the velocity field in a one-dimensional flow, where the members are obtained from measurements at $M$ different times, or can represent ensemble averages 
over $M$ distinct experiments [2]. The objective is to find a basis $\left\{\phi_{j}(x)\right\}_{j=1}^{\infty}$ in a Hilbert space with inner product that is $\mathcal{L}_{2}$ optimal. That is, a basis is sought such that the error made by the approximation of order $r$ :

$$
w(x) \approx w_{r}(x)=\sum_{j=1}^{r} a_{j} \phi_{j}(x),
$$

is the smallest among those achieved with other bases and same order. The problem can be formally stated as:

$$
\min _{\phi}\left\langle\left\|w-\frac{(w, \phi)}{\|\phi\|^{2}} \phi\right\|^{2}\right\rangle
$$

where $\langle\cdot\rangle$ denotes averaging over the members, $(\cdot, \cdot)$ is an inner product, and $\|f\|=(f, f)^{\frac{1}{2}}$. The choice of inner product is a very important step and can be informed by prior knowledge of the problem domain. A common choice is to search for the basis in $\mathbb{R}^{n}$ and take $(x, y)=y^{\top} x$. In this case, the optimal basis is given by the eigenvectors $\left\{\phi_{j}\right\}_{j=1}^{n}$ of the correlation matrix $\mathcal{R}=$ $\frac{1}{M} \sum_{k=1}^{M} w_{k} w_{k}^{\top} \in \mathbb{R}^{n \times n}$, called POD modes. Moreover, the eigenvalues of $\mathcal{R}$, denoted by $\lambda_{j}$ and always real nonnegative, are proportional to the energy captured in the associated mode $\phi_{j}$. This has the important implication that (assuming the eigenvalues have been ordered by $\left.\lambda_{j} \geq \lambda_{j+1}\right)$ the subspace of order $r \operatorname{span}\left(\phi_{[1, r]}\right)$ captures the fraction $\frac{\sum_{j=1}^{r} \lambda_{j}}{\sum_{j=1}^{n} \lambda_{j}}$ of total energy in the observed data. From a computational viewpoint, the eigendecomposition of $\mathcal{R}$ is costly because $n \gg 1$. This can be overcome by noting that $\left(\phi_{j}, \lambda_{j}\right)$ can also be obtained from the SVD of $w_{[1, M]}$ :

$$
\begin{gathered}
w_{[1, M]}=U \Sigma V^{\top} \\
\sigma_{j}=\Sigma(j, j) \quad \lambda_{j}=\frac{\sigma_{j}^{2}}{M}, \quad \phi_{j}=U(:, j) .
\end{gathered}
$$

Dimensionality reduction is achieved by using the projection operator $S_{r}=\phi_{[1, r]} \phi_{[1, r]}^{\top}$ which maps $\mathbb{R}^{n}$ into the subspace $Q$ of size $r<n$ spanned by $\phi_{[1, r]}=: P_{r}$.

While POD has proved successful in producing loworder dynamical models, a recognized drawback is that the subspace $Q$ does not account for dynamical effects, and the optimality criterion (4) might not always lead to accurate results. The Dynamic Mode Decomposition (DMD) technique was proposed in [3] to partially address these aspects, and can be understood as a method to compute a low-order eigendecomposition of a linear operator which describes the evolution of a given sequence of observations [13]. The key step is to project the data onto a lower dimensional subspace where a linear regression can be efficiently computed. Even though researchers have looked at improved strategies for the data projection step (e.g. optimization over manifolds techniques [14]), the standard choice is an orthogonal projection onto the subspace $Q$ spanned by the first $r$ POD modes associated with the sequence of computed states.
While initial works focused on autonomous systems, recent extensions of the DMD framework to controlled systems (DMDc) [4] and input-output models (IODMD) [5] have been proposed, all using $Q$ as subspace for the projection. If input $u_{[1, M]}$, state $x_{[1, M]}$, and output $y_{[1, M]}$ trajectories are available, a model of order $r$ can then be estimated by solving the least-squares problem:

$$
\min _{A, B, C, D}\left\|\left[\begin{array}{c}
\bar{x}_{[2, M]} \\
y_{[1, M-1]}
\end{array}\right]-\left[\begin{array}{cc}
A & B \\
C & D
\end{array}\right]\left[\begin{array}{l}
\bar{x}_{[1, M-1]} \\
u_{[1, M-1]}
\end{array}\right]\right\|_{F}^{2},
$$

where $\bar{x}_{[1, M]}=P_{r}^{\top} x_{[1, M]} \in \mathbb{R}^{r \times M}$, and $P_{r} \in \mathbb{R}^{n \times r}$ is made of the leading $r$ singular vectors of the so-called snapshot matrix $x_{[1, M-1]}$.

\section{EXTRACTING STATES FROM DATA}

It is clear that the SId algorithm reviewed in Section IIA (related by a unifying theory to other SId approaches [7]) and DMD feature important similarities. In both cases, the model estimation step boils down to a leastsquares fit (3)-(5) done on input, state, and output sequences. A fundamental role is played by the state, which in both cases is a proxy of the true one. In SId, the state is not measured and a particular sequence is extracted from the intersection between two subspaces built from input-output trajectories. In DMD there is full observation, and the state $\bar{x}$ used for fitting is obtained by projecting the high-dimensional state onto the subspace spanned by the POD modes (computed as well from the states). Owing to these modes' aforementioned property, the state components are here ranked by energy content, paving the way to the use of DMD as a model reduction technique. Similarly, truncating SVD factorizations is in subspace identification a common heuristics for obtaining reduced-order models and denoising the data. However, this is not underpinned by a clear rationale, for example on the relevance of the states that are neglected. The next sections provide results that shed light into the selection of particular state sequences in SId and DMD techniques.

\section{A. Parametrized POD coordinate transformations}

It is known that state-space models such as (1) are only defined up to a similarity transformation. While many important properties of the LTI system remains invariant under this transformation, the state does not. Specifically, given a state $x, \tilde{x}=T x$ also qualifies as state for any nonsingular matrix $T \in \mathbb{R}^{n_{x} \times n_{x}}$ defining a particular coordinate transformation, which only leaves the state range space unchanged, i.e. $\operatorname{span}\left(\tilde{x}_{[i, j]}\right)=\operatorname{span}\left(x_{[i, j]}\right)$. A similarity transformation which provides a common ground between DMD and SId is defined next (it is assumed that $j-i+1>n_{x}$, which is necessary in SId).

Definition 1: Given a state sequence $x_{[i, j]}$, a nonsingular matrix $T_{\mathrm{POD}}$ defines a POD transformation if:

$$
\tilde{x}_{[i, j]}=T_{\mathrm{POD}} x_{[i, j]}=\tilde{\Sigma} \tilde{V}^{\top},
$$

where $\tilde{\Sigma} \in \mathbb{R}^{n_{x} \times n_{x}}$ is any positive definite diagonal matrix (with entries in decreasing order) and

$\tilde{V} \in \mathbb{R}^{(j-i+1) \times n_{x}}$ is any matrix with orthogonal columns. 
Essentially, $T_{\mathrm{POD}}$ defines a coordinate frame where states are ranked by their energy content. Obviously, for a given state sequence $x_{[i, j]}$ POD transformations are nonunique. Next lemma gives a parametrization of POD coordinate transformations.

Lemma 1: Given a state sequence $x_{[i, j]}=U \Sigma V^{\top}$, then any nonsingular matrix $\tilde{T}_{\mathrm{POD}}$ :

$$
\tilde{T}_{\mathrm{POD}}=\tilde{\Sigma} \hat{V}^{\top} \Sigma^{\dagger} U^{\top},
$$

where $\tilde{\Sigma} \in \mathbb{R}^{n_{x} \times n_{x}}$ is any positive definite diagonal matrix and $\hat{V} \in \mathbb{R}^{n_{x} \times n_{x}}$ is any orthogonal matrix, defines a POD transformation with:

$$
\tilde{x}_{[i, j]}=\tilde{T}_{\mathrm{POD}} x_{[i, j]}=\tilde{\Sigma} \tilde{V}^{\top}, \quad \tilde{V}=V \hat{V}
$$

Proof: From Definition 1 and orthogonality of the left singular vectors $U$, it is clear that a candidate $T_{\mathrm{POD}}$ is simply $T_{\mathrm{POD}}=U^{\top}$. This would give:

$$
\tilde{x}_{[i, j]}=T_{\mathrm{POD}} x_{[i, j]}=\Sigma V^{\top}
$$

and is such that $\tilde{x}_{[i, j]}$ and $x_{[i, j]}$ have the same singular values. However, any matrix $\tilde{T}_{\mathrm{POD}}$ which satisfies:

$$
\begin{aligned}
\tilde{T}_{\mathrm{POD}} T_{\mathrm{POD}}^{\top} \Sigma & =\tilde{\Sigma} \hat{V}^{\top}, \\
\tilde{T}_{\mathrm{POD}} & =\tilde{\Sigma} \hat{V}^{\top} \Sigma^{\dagger} U^{\top},
\end{aligned}
$$

for arbitrary positive definite diagonal and orthogonal matrices, respectively $\tilde{\Sigma}$ and $\hat{V}$, would give a new state sequence in POD coordinates:

$$
\tilde{x}_{[i, j]}=\tilde{T}_{\mathrm{POD}} x_{[i, j]}=\tilde{\Sigma} \hat{V}^{\top} \Sigma^{\dagger} U^{\top} U \Sigma V^{\top}=\tilde{\Sigma}(\underbrace{V \hat{V}}_{\tilde{V}})^{\top},
$$

where $\tilde{V} \in \mathbb{R}^{(j-i+1) \times n_{x}}$ is the product of a matrix with orthogonal columns by an orthogonal matrix and thus has orthogonal columns.

Remark 1: Eq. (7) shows that POD transformations can be parametrized by choosing the $n_{x}$ singular values $\tilde{\Sigma}$ of the projected state $\tilde{x}_{[i, j]}$ (singular values placement). This non-uniqueness is not surprising, and relates to the fact that the original state sequence $x_{[i, j]}$ is not uniquely defined. In DMD algorithms, the state $\bar{x}$ is determined using the particular transformation (9) which preserves the singular values of the original sequence $x_{[i, j]}$. However, these clearly depend on the similarity transformation underlying (1). In fact, using (7) one can readily obtain a similarity transformation for the original sequence such that, after applying the standard transformation (9), the states are ranked according to the (arbitrary) singular values in $\tilde{\Sigma}$. This is believed to have strong ties with the selection of inner product (4) in the definition of POD modes, known from the early works [2] to have a very important role, but largely overlooked in applications.

A natural question is then in which coordinate system is the state $\hat{x}$ extracted by the subspace algorithm.

Lemma 2: Given matrices $H_{1,2 l}$ and $H_{2,2 l}$ (2) built from persistently exciting input and noise-free output trajectories $u_{[1, N]}$ and $y_{[1, N]}$, define the following SVD and QR factorizations:

$$
\begin{aligned}
H=\left[\begin{array}{l}
H_{1,2 l} \\
H_{2,2 l}
\end{array}\right] & =\left[\begin{array}{ll}
U_{11} & U_{12} \\
U_{21} & U_{22}
\end{array}\right]\left[\begin{array}{cc}
\Sigma_{11} & 0 \\
0 & 0
\end{array}\right]\left[\begin{array}{ll}
V_{1} & V_{2}
\end{array}\right]^{\top} \\
U_{12}^{\top} U_{11} \Sigma_{11} & =U_{x} \Sigma_{x} V_{x}^{\top} \\
U_{x}^{\top} & =Q_{x} R_{x} .
\end{aligned}
$$

Then, $Q_{x}$ defines a POD transformation for the extracted state sequence $x_{[l+1, N-l+1]}$ :

$$
\begin{aligned}
& x_{[l+1, N-l+1]}=R_{x} U_{12}^{\top} H_{1,2 l}, \\
& \hat{x}_{[l+1, N-l+1]}=Q_{x} x_{[l+1, N-l+1]}=\Sigma_{x}\left(V_{1} V_{x}\right)^{\top} .
\end{aligned}
$$

Proof: The SVD (10a) identifies the order of the system (since $\left.\Sigma_{11} \in \mathbb{R}^{\left(2 \ln _{u}+n_{x}\right) \times\left(2 \ln _{u}+n_{x}\right)}\right)$ and the orthogonal complement $\left[\begin{array}{l}U_{12} \\ U_{22}\end{array}\right]$, which in turn reveals the intersection between the range spaces of $H_{1,2 l}$ and $H_{2,2 l}$ :

$$
U_{12}^{\top} H_{1,2 l}=-U_{22}^{\top} H_{2,2 l} \in \mathbb{R}^{\left(2 l n_{y}-n_{x}\right) \times N-2 l+1},
$$

As proven in [8], the intersection subspace has only $n_{x}$ independent vectors, coinciding with a valid state sequence. To find a basis for it, observe using (10a) that:

$$
U_{12}^{\top} H_{1,2 l}=\left[U_{12}^{\top} U_{11} \Sigma_{11}\right] V_{1}^{\top}
$$

and hence $\operatorname{rank}\left(U_{12}^{\top} U_{11} \Sigma_{11}\right)=n_{x}$. Therefore, using the factorizations (10b)-(10c), it holds:

$$
\begin{aligned}
& R_{x} U_{12}^{\top} H_{1,2 l}=R_{x} U_{x} \Sigma_{x}\left(V_{1} V_{x}\right)^{\top}=x_{[l+1, N-l+1]}, \\
&Q_{x} \underbrace{}_{x_{[l+1, N-l+1]} R_{x} U_{12}^{\top} H_{1,2 l}}=\underbrace{\Sigma_{x}}_{\tilde{\Sigma}} \underbrace{V_{1} V_{x}}_{\tilde{V}})^{\top}=\hat{x}_{[l+1, N-l+1]},
\end{aligned}
$$

which proves the statement.

The algorithm in [8] also employs the SVD (10a)(10b), but their implications on the state sequence coordinate definition were not addressed. Lemma 2 proves two things: the states $\hat{x}_{[l+1, N-l+1]}$ from the intersection algorithm are in POD coordinates; and the particular POD transformation used here is the one assigning to the extracted state sequence the non-zero singular values of $U_{12}^{\top} H_{1,2 l}$ (i.e. the entries of $\Sigma_{x}$ ). It is worth observing that, since the latter matrix depends on input-output data only, the particular POD transformation matrix used here $Q_{x}$ is not affected by the arbitrariness related to similarity transformations. Moreover, in SId the calculation of $T_{\mathrm{POD}}$ is done in advance (up-front), that is, the transformation is not computed from, but together with, the state sequence. This is contrast with DMD methods where the computation of the POD modes requires knowledge of the state sequence $x_{[l+1, N-l+1]}$. Note also that SVD (10b), enabling the subspace-POD projection, has a computational complexity depending only on the depth $2 l$. Instead, the computation of POD modes depends on the data length, which is potentially onerous if the sequence is long. 


\section{B. Estimating balanced states from data}

It has been shown earlier that both DMD and SId methods use states ranked by energy content in their respective least-squares problems, thus providing a means for obtaining models of reduced order $r$ by removing the last $n_{x}-r$ states. This strategy is however questioned by the arbitrariness of $T_{\mathrm{POD}}$, unless one has a precise rationale to select a specific $\mathrm{POD}$ transformation out of the infinitely many defined by Lemma 1 . It is therefore of interest to consider alternative similarity transformations. For example, the balanced one, as it is central in model order reduction of LTI systems.

Definition 2: [15] A nonsingular matrix $T_{\text {Bal }}$ defines a balanced transformation for (1) if:

$$
T_{\text {Bal }}=\Sigma_{b}^{-0.5} U_{b}^{\top} L_{o}^{\top},
$$

where $L_{o}$ and $L_{r}$ are the Cholesky factors of the Gramians $W_{o}$ and $W_{r}$, respectively, and $L_{o}^{\top} L_{r}=U_{b} \Sigma_{b} V_{b}^{\top}$.

When the singular values of the product $W_{r} W_{o}$ (the Hankel singular values) are distinct, $T_{\text {Bal }}$ is unique to within the sign of its columns. This transformation diagonalizes (with same diagonal entries, equal to the Hankel singular values) $W_{r}$ and $W_{o}$, and the resulting states are ranked based on their degree of controllability/observability. Balanced truncation then consists of removing the states associated with the smallest $n_{x}-r$ Hankel singular values, justified by the fact that their sum provides a lower bound and, for systems in balanced coordinates, an upper bound on the quality of the approximation [16]. Even though not guaranteed to be optimal, balanced truncation is de facto a standard tool in model-based order reduction.

The finite time- $l$ observability and reachability Gramians [17] are defined respectively as $W_{o, l}=\Gamma_{l}^{\top} \Gamma_{l}$ and $W_{r, l}=\Omega_{l} \Omega_{l}^{\top}$. When they are used, instead of $W_{o}$ and $W_{r}$, to build the transformation (14), the state sequence is said to be in finite time- $l$ balanced coordinates. While the error bounds available for the infinite-time balanced case do not hold in this case, by making $l$ large enough (how large depends on the impulse response decay rate) the two transformations can be made arbitrarily close [10]. In the remainder it is assumed that $l$ is chosen such that the approximation introduced by the finite timeGramians is negligible.

The use of balanced truncation in the context of DMD methods has been recently suggested in [18] to replace the orthogonal projection onto the POD modes by an oblique projection onto empirical balancing modes. This is achieved at the cost of additional ad-hoc simulations to construct the finite time-Gramians [19]. The SId framework instead allows one to directly extract a balanced state sequence using only input output trajectories [9], [10]. This is presented in the following Lemma, which builds on the original result from [9] (variables already introduced in Lemma 2 are used with same notation and the subscript $2 l$ is dropped for readability). We first define the following partition of the orthogonal complement of $H$, which corresponds to the input-output parts in $H_{1}$ and $H_{2}$, i.e. $\left[\begin{array}{ll}U_{12}^{\top} & U_{22}^{\top}\end{array}\right]=\left[\begin{array}{llll}P_{1, u} & P_{1, y} & P_{2, u} & P_{2, y}\end{array}\right]$.

Lemma 3: Given trajectories $u_{[1, N]}$ and $y_{[1, N]}$, it holds:

$$
\begin{aligned}
\Gamma_{l} & =P_{2, y}^{\dagger} R_{x}^{\top}, \\
\Gamma_{l} \Omega_{l}=P_{2, y}^{\dagger}\left(P_{1, y} P_{2, y}^{\dagger} P_{2, u}-P_{1, u}\right) & =U_{T} \Sigma_{T} V_{T}^{\top}, \\
\hat{T}_{x} & =\Sigma_{T}^{-0.5} U_{T}^{\top} \Gamma_{l} .
\end{aligned}
$$

That is, matrices derived from data can be used to explicitly construct the extended observability matrix $\Gamma_{l}$ and the matrix $\hat{T}_{x}$ projecting the state sequence $x_{[l+1, N-l+1]}$ (11a) in finite time- $l$ balanced coordinates.

Proof: In Lemma 2 it was proved that $x_{[l+1, N-l+1]}$ is a valid state sequence (in generic coordinates). The proof here uses this as starting point, and constructs the balancing transformation $\hat{T}_{x}$ for the representation associated with $x_{[l+1, N-l+1]}$ in three steps.

The input-output equations of (1) yields:

$$
\begin{array}{r}
H_{2}(l+1: 2 l,:)=\Gamma_{l} x_{[l+1, N-l+1]}+\mathcal{T}_{l} H_{2}(1: l,:), \\
\rightarrow x_{[l+1, N-l+1]}=\left[\begin{array}{ll}
-\Gamma_{l}^{\dagger} \mathcal{T}_{l} & \Gamma_{l}^{\dagger}
\end{array}\right] H_{2},
\end{array}
$$

The sequence $x_{[l+1, N-l+1]}$ can also be written using (11a). Substituting (12) in (11a), the two equivalent expressions for the state sequence give:

$$
\left[\begin{array}{ll}
-\Gamma_{l}^{\dagger} \mathcal{T}_{l} & \Gamma_{l}^{\dagger}
\end{array}\right] H_{2}=R_{x}\left[\begin{array}{ll}
P_{2, u} & P_{2, y}
\end{array}\right] H_{2},
$$

This relationship, observing that $R_{x}$ has orthonormal columns, can be used to construct the extended observability matrix and thus proves (15a).

The second step consists of proving (15b), for which the same arguments in [9] are followed. From the inputoutput equations it also holds:

$$
H_{1}(l+1: 2 l,:)=\Gamma_{l} x_{[1, N-2 l+1]}+\mathcal{T}_{l} H_{1}(1: l,:),
$$

Pre-multiplying (16) and (18) by the orthogonal complement of $\Gamma_{l}$ (denoted by $\Gamma_{l}^{\perp}$ ) yields:

$$
0=\left[\begin{array}{ll}
-\Gamma_{l}^{\perp} \mathcal{T}_{l} & \Gamma_{l}^{\perp}
\end{array}\right] H_{1}=\left[\begin{array}{ll}
-\Gamma_{l}^{\perp} \mathcal{T}_{l} & \Gamma_{l}^{\perp}
\end{array}\right] H_{2},
$$

Using the fact that the state sequence is in the span of $U_{22}^{\top} H_{2}$, and eqs.(17)-(19), it is possible to write:

$$
\left[\begin{array}{ll}
P_{2, u} & P_{2, y}
\end{array}\right]=R_{x}^{\top}\left[\begin{array}{ll}
-\Gamma_{l}^{\dagger} \mathcal{T}_{l} & \Gamma_{l}^{\dagger}
\end{array}\right]+\mathcal{B}\left[\begin{array}{ll}
-\Gamma_{l}^{\perp} \mathcal{T}_{l} & \Gamma_{l}^{\perp}
\end{array}\right],
$$

where $\mathcal{B}$ is any arbitrary matrix of appropriate size. Then, an equivalent formula for $U_{12}^{\top}$ is obtained by observing that $x_{[l+1,2 N-l+1]}=A^{l} x_{[1,2 N-2 l+1]}+\Omega_{i} H_{1}(1$ : $l,:)$, and by using eqs.(12-19):

$$
\begin{aligned}
{\left[\begin{array}{ll}
P_{1, u} & P_{1, y}
\end{array}\right]=} & R_{x}^{\top}\left[\left(\begin{array}{ll}
\left.A^{l} \Gamma_{l}^{\dagger} \mathcal{T}_{l}-\Omega_{l}\right) & -A^{l} \Gamma_{l}^{\dagger}
\end{array}\right]+\right. \\
& +\mathcal{C}\left[\begin{array}{ll}
-\Gamma_{l}^{\perp} \mathcal{T}_{l} & \Gamma_{l}^{\perp}
\end{array}\right],
\end{aligned}
$$

where again $\mathcal{C}$ is any arbitrary matrix of appropriate size. Eqs. (20) and (21) finally give:

$$
\begin{aligned}
P_{1, y} P_{2, y}^{\dagger} P_{2, u}-P_{1, u} & =-P_{1, y} \mathcal{T}_{l}-P_{1, u}=R_{x}^{\top} \Omega_{l}, \\
P_{2, y}^{\dagger} & =\left[\begin{array}{ll}
\Gamma & \Gamma^{\perp \dagger}
\end{array}\right]\left[\begin{array}{ll}
R_{x} & \mathcal{B}
\end{array}\right]^{\dagger},
\end{aligned}
$$


which, together with (15a), prove (15b).

Finally, given an expression for $\Gamma_{l} \Omega_{l}$, it is possible to show that the matrix $\hat{T}_{x}$ in $(15 \mathrm{c})$ provides a balanced state sequence. Recalling the SVD (15b), it holds:

$$
\begin{gathered}
\underbrace{\left(\Sigma_{T}^{-0.5} U_{T}^{\top} \Gamma_{l}\right)}_{\hat{T}_{x}} W_{r, l}\left(\Sigma_{T}^{-0.5} U_{T}^{\top} \Gamma_{l}\right)^{\top}=\Sigma_{T}, \\
\left(\Omega_{l} V_{T} \Sigma_{T}^{-0.5}\right)^{\top} W_{o, l} \underbrace{\left(\Omega_{l} V_{T} \Sigma_{T}^{-0.5}\right)}_{\hat{T}_{x}^{-1}}=\Sigma_{T} .
\end{gathered}
$$

That is, $\hat{T}_{x}$ diagonalizes (with same diagonal) the Gramians, hence is the sought balanced transformation.

Lemma 3 provides new elements with respect to the original result in [9]. The proof is linked with that of Lemma 2 , and by doing so the connection with the sequence of states in POD coordinates is made. Moreover, an explicit expression for the transformation $\hat{T}_{x}$ is obtained here, while in [9] only the reduction (fat) matrix that gives the state via multiplication by $U_{12}^{\top} H_{1}$ was given.

An important fact is that, similarly to what was observed earlier for POD states, in SId the balanced transformation is performed up-front. This is an interesting fact considering that there is no direct access to the state, while in DMD, despite having full observability, further ad-hoc simulations would be required [18]. The reason for this is that subspace identification favourably exploits the structural properties of the underlying linear system to extract states (and as a result state-space models) in the desired coordinates. This points out the potential of investigating balancing projections in DMD which only require input-output matrices.

\section{NumericAl EXAMPLES}

\section{A. An illustrative non-normal system}

Consider the SISO system defined as:

$$
\begin{gathered}
A:=\left\{\begin{array}{l}
A_{i j}=0.2, \quad i=j \\
A_{i j}=2.5, \quad i>j \\
A_{i j}=0, \quad i<j
\end{array} \quad \in \mathbb{R}^{10 \times 10},\right. \\
B=\left[\begin{array}{lll}
1 \ldots 1
\end{array}\right] \in \mathbb{R}^{10}, \quad C=c\left[\begin{array}{lll}
1 & \ldots & 1
\end{array}\right]^{\top} \in \mathbb{R}^{1 \times 10}, \quad D=0 .
\end{gathered}
$$

where $c$ is a normalization factor such that the system has unit DC gain, and the sampling time is $T_{s}=0.03 \mathrm{~s}$. The upper-triangular structure of $A$ makes this system non-normal, that is $A$ does not commute with its adjoint and thus its eigenvectors are not orthogonal (in fact, they are all parallel here). An important consequence of this is that, even if the system is stable, its unforced response does not decrease monotonically and can exhibit large transient growth before eventually decaying. This type of systems are often encountered in fluid mechanics problems (e.g. linearized Navier-Stokes equations), see for example [11].

The impulse response of the reduced-order models obtained by removing in the least-squares problems the smallest $n_{x}-r$ states are examined. Specifically, results are compared in Figure 1 for: IODMD (problem in Eq. 5 with states obtained by projecting the simulated states onto the first $r$ POD modes); SId-POD (problem in Eq. 3 with states extracted directly in POD coordinates, as per Eq. 11b); and SId-Bal (problem in Eq. 3 with states extracted directly in balanced coordinates). The sensitivity of IODMD to the state coordinate of the original model is also investigated by generating a sequence of states from the companion form of (23) (IODMD-comp). The analyses are performed with $N=510, M=N, l=20$, and using as the input a PRBS-9 sequence [1].
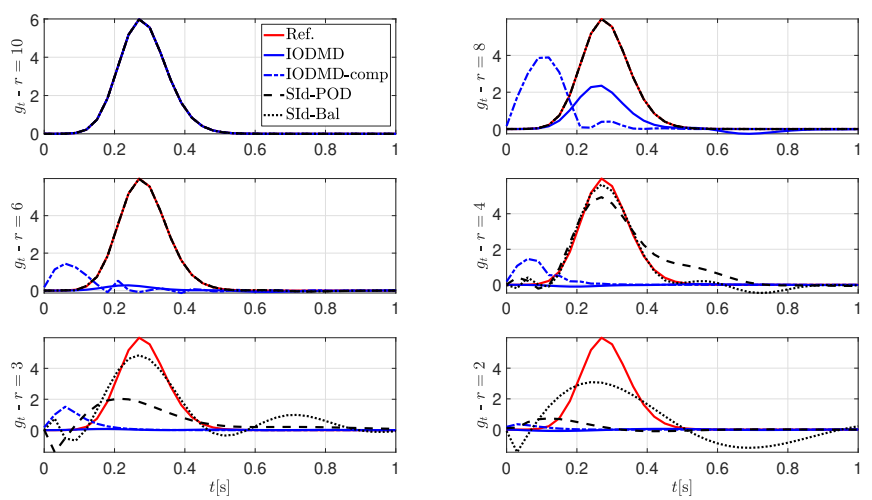

Fig. 1: Impulse response of the estimated models with full-order $(r=10)$ and reduced-orders $(r=8,6,4,3,2)$.

The impulse response of (23), reported for reference (Ref.), shows the non-monotonicity characteristic of nonnormal systems. It is noted that, as $r$ decreases, the quality of the model estimated by the IODMD approach markedly deteriorates (note that for $r<8$ the transient feature is not even qualitatively captured). Moreover, a big difference is noted between IODMD and IODMDcomp. Note that while the choice of companion form is fictitious here, the arbitrariness of the similarity transformation can happen in practice in high-order models (a trivial but often encountered example is the normalization of state variables by arbitrary reference quantities). The SId-Bal estimates capture dominant features of the original impulse response also for very low $r$. It is also worth observing that SId-POD performs considerably better than $I O D M D$, despite the fact that both methods remove states based on their energy contents.

\section{B. A thermoacoustic test-case}

In the second example, a model describing thermoacoustic effects in the Rijke tube system is considered [12]. The dynamics is described by nonlinear partial differential equations consisting of continuity and momentum balances, which can be linearized about a specified trim point and then converted into ordinary differential equations by means of spatial discretization schemes. This is done here by projecting the dynamics onto Galerkin modes, which are sinusoidal functions of the position inside the Tube with harmonically related frequencies, 
see [20] for details. The autonomous model proposed in the reference is augmented here to account for input (modelled as in boundary condition control) and output (representing sensor measurements) channels. The final model, used to generate the data, consists of 200 states (each Galerkin mode is associated with two states), 1 input modeling the action of a speaker at the bottom of the tube, and 1 output consisting of the pressure measured at $80 \%$ of the length. The analyses are performed with $N=1022, M=N, l=270$, and using as input a PRBS10 sequence, a type of input also used in [21] for real experiments on the Rijke tube. However, other inputs such as chirp could also be considered.

Figure 2 shows two relative error metrics between the original and estimated frequency response's magnitude, namely $e_{2}=\frac{\sum_{i=1}^{n \omega}\left|G\left(e^{j \omega_{i}}\right)-\hat{G}_{r}\left(e^{j \omega_{i}}\right)\right|^{2}}{\sum_{i=1}^{n_{\omega}}\left|G\left(e^{j \omega_{i}}\right)\right|^{2}}$ (left plot) and $e_{\infty}=\frac{\max _{\omega_{i}}\left|G\left(e^{j \omega_{i}}\right)-\hat{G}_{r}\left(e^{j \omega_{i}}\right)\right|}{\max _{\omega_{i}}\left|G\left(e^{j \omega_{i}}\right)\right|}$ (right plot), on $n_{\omega}=200$ frequency points for different values of model order $r$.
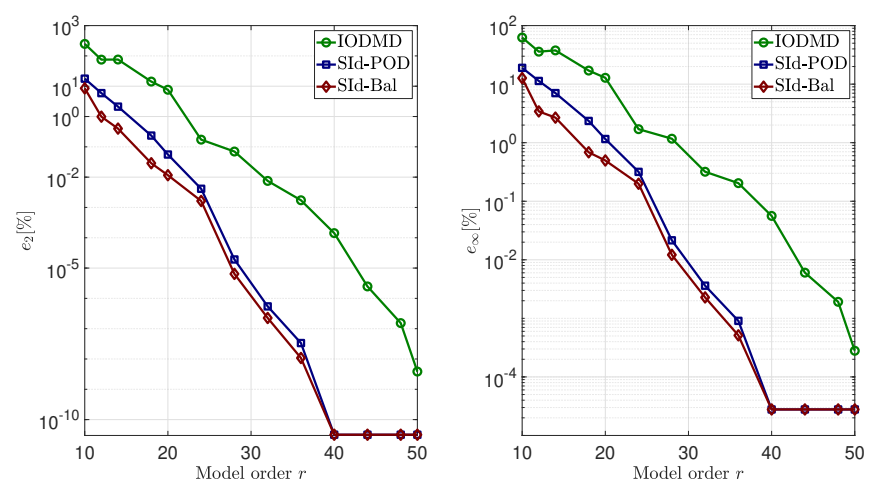

Fig. 2: Percentage relative errors $\left(e_{2}\right.$-left, $e_{\infty}$-right $)$ of the frequency response as a function of the model's order.

The results reinforce the evidence gathered in the previous example and are obtained considering a physical system which is representative of a typical application of DMD techniques [22]. The plotted trend confirms that subspace identification with balanced states outperform the other algorithms when seeking low-order approximations. Moreover, it is confirmed that the particular POD transformation selected in the subspace identification method outperforms the one adopted in IODMD (computed from the high-dimensional state sequence). This inherent robustness was observed also in other numerical tests and general arguments that underpin its success deserve further investigations.

\section{Conclusions}

Similarities and important distinctions between subspace identification and Dynamic Mode Decomposition approaches with focus on the use of fictitious state sequences are formally presented. A parametrization of POD state transformations is derived and it is shown how the approaches can be interpreted as two different choices of this transformation. New insights into a balancing transformation only requiring input-output trajectories are also described. The results corroborate the main claim that the standard POD projection step employed in DMD approaches might not be robust to general applications since the quality of the estimated model strongly depends on the particular state sequence considered. This emphasizes the importance of selecting an appropriate inner product when using POD.

\section{REFERENCES}

[1] L. Ljung, System Identification: Theory for the User. Prentice Hall PTR, 1999.

[2] P. Holmes, J. L. Lumley, G. Berkooz, and C. W. Rowley, Turbulence, Coherent Structures, Dynamical Systems and Symmetry, 2nd ed. Cambridge University Press, 2012.

[3] P. Schmid, "Dynamic mode decomposition of numerical and experimental data," Journal of Fluid Mechanics, vol. 656, pp. 5-28, 2010.

[4] J. L. Proctor, S. L. Brunton, and J. N. Kutz, "Dynamic mode decomposition with control," SIAM Journal on Applied Dynamical Systems, vol. 15, no. 1, pp. 142-161, 2016.

[5] J. Annoni and P. Seiler, "A method to construct reduced-order parameter-varying models," International Journal of Robust and Nonlinear Control, vol. 27, no. 4, pp. 582-597, 2017.

[6] P. van Overschee and B. de Moor, Subspace Identification for Linear Systems: Theory, Implementation, Applications. Kluwer Academic Publishers, 1996.

[7] P. Van Overschee and B. De Moor, "A unifying theorem for three subspace system identification algorithms," Automatica, vol. 31, no. 12, pp. 1853 - 1864, 1995.

[8] M. Moonen, B. D. Moor, L. Vandenberghe, and J. Vandewalle, "On- and off-line identification of linear state-space models," International Journal of Control, vol. 49, no. 1, pp. 219-232, 1989.

[9] M. Moonen and J. Ramos, "A subspace algorithm for balanced state space system identification," IEEE Transactions on Automatic Control, vol. 38, no. 11, pp. 1727-1729, 1993.

[10] I. Markovsky, J. C. Willems, P. Rapisarda, and B. L. De Moor, "Algorithms for deterministic balanced subspace identification," Automatica, vol. 41, no. 5, pp. 755 - 766, 2005.

[11] P. Schmid and D. Henningson, Stability and Transition in Shear Flows. Springer-Verlag New York, 2001.

[12] J. P. Epperlein, B. Bamieh, and K. J. Astrom, "Thermoacoustics and the Rijke Tube: Experiments, Identification, and Modeling," IEEE Control Systems Magazine, vol. 35, no. 2, pp. 57-77, 2015.

[13] J. H. Tu, C. W. Rowley, D. N. Luchtenburg, S. L. Brunton, and J. N. Kutz, "On dynamic mode decomposition: Theory and applications," Journal of Computational Dynamics, vol. 52, no. 1, pp. 477-508, 2014.

[14] P. J. Goulart, A. Wynn, and D. Pearson, "Optimal mode decomposition for high dimensional systems," in IEEE Conference on Decision and Control, 2012.

[15] A. Laub, M. Heath, C. Paige, and R. Ward, "Computation of system balancing transformations and other applications of simultaneous diagonalization algorithms," IEEE Transactions on Automatic Control, vol. 32, no. 2, pp. 115-122, 1987.

[16] K. Glover, "All optimal Hankel-norm approximations of linear multivariable systems and their $L_{\infty}$-error bounds," International Journal of Control, vol. 39, no. 6, pp. 1115-1193, 1984.

[17] A. C. Antoulas, Approximation of Large-Scale Dynamical Systems. Society for Industrial and Applied Mathematics, 2005.

[18] A. Iannelli, U. Fasel, and R. Smith, "The Balanced Mode Decomposition Algorithm for Data-Driven LPV Low-Order Models of Aeroservoelastic Systems," Aerospace Science and Technology (in press), 2021. 
[19] S. Lall, J. E. Marsden, and S. Glavaški, "A subspace approach to balanced truncation for model reduction of nonlinear control systems," International Journal of Robust and Nonlinear Control, vol. 12, no. 6, pp. 519-535, 2002.

[20] M. Juniper, "Triggering in the horizontal rijke tube: nonnormality, transient growth and bypass transition," Journal of Fluid Mechanics, vol. 667, pp. 272-308, 2011.

[21] A. Iannelli, M. S. Baumann, S. Balula, and R. S. Smith, "Experiments and identification of thermoacoustic instabilities with the Rijke tube," in IEEE Conference on Control Technology and Application, 2020.

[22] T. Sayadi, V. Le Chenadec, P. Schmid, F. Richecoeur, and M. Massot, "Thermoacoustic instability - a dynamical system and time domain analysis," Journal of Fluid Mechanics, vol. 753, pp. 448-471, 2014 